\title{
Mobile Sensing Around the Globe: Considerations for Cross-Cultural Research
}

\author{
Le Vy Phan ${ }^{\dagger 1}$, Nick Modersitzki ${ }^{\dagger 1}$, Kim K. Gloystein ${ }^{1}$, Sandrine R. Müller ${ }^{1, *}$ \\ ${ }^{1}$ Department of Psychology, Bielefeld University, Germany
}

Version: January 5, 2022

This chapter will be published in a book on Technology and Measurement Around the Globe by Cambridge University Press.

This preprint may differ from the final, copy-edited publication.

\author{
Author Note \\ †These authors have contributed equally. \\ * Present address: Google LLC, New York, NY, USA
}

Le Vy Phan ORCID iD: https://orcid.org/0000-0002-6470-8871

Nick Modersitzki ORCID iD: https://orcid.org/0000-0003-4803-806X

Kim K. Gloystein ORCID iD: https://orcid.org/0000-0001-6235-067X

Sandrine R. Müller ORCID iD: https://orcid.org/0000-0002-1226-6370

All authors declare they have no competing interests. We thank Aaron Cohen, Namrata Goyal, Seth Margolis, and John Rauthmann for feedback on earlier versions of this manuscript. Correspondence concerning this book chapter should be addressed to Le Vy Phan and Nick Modersitzki, Abteilung Psychologie, Universität Bielefeld, Universitätsstraße 25, D-33615 Bielefeld, Germany. Email: le.phan@uni-bielefeld.de; nick.modersitzki@uni-bielefeld.de 


\begin{abstract}
The ubiquity of mobile devices allows researchers to assess people's real-life behaviors objectively, unobtrusively, and with high temporal resolution. As a result, psychological mobile sensing research has grown rapidly. However, only very few cross-cultural mobile sensing studies have been conducted to date. In addition, existing multi-country studies often fail to acknowledge or examine possible cross-cultural differences. In this chapter, we illustrate biases that can occur when conducting cross-cultural mobile sensing studies. Such biases can relate to measurement, construct, sample, device type, user practices, and environmental factors. We also propose mitigation strategies to minimize these biases, such as the use of informants with expertise in local culture, the development of cross-culturally comparable instruments, the use of culture-specific recruiting strategies and incentives, and rigorous reporting standards regarding the generalizability of research findings. We hope to inspire rigorous comparative research to establish and refine mobile sensing methodologies for cross-cultural psychology.
\end{abstract}

Keywords: Mobile sensing, cross-cultural research, measurement bias, measurement invariance, mitigation strategies 


\section{Mobile Sensing Around the Globe: Considerations for Cross-Cultural Research}

Smartphone usage around the globe has accelerated throughout the 2010s. As of 2020, nearly $45 \%$ of the world's population owns a smartphone, and this percentage is expected to continue rising in the coming years (Statista, 2020). The ubiquity of mobile devices presents numerous possibilities for the use of mobile sensing. Mobile sensing methods (MSMs) make use of the multitude of sensors embedded in mobile devices and wearables to collect and process data about people's behavior and their environments (Khan et al., 2013). The ability to collect large-scale behavioral data based on people's everyday lives in a granular, continuous, and dynamic manner has inspired a wave of psychological mobile sensing research (see, e.g., Harari et al., 2016). Mobile sensing data have been used in psychological research to examine social interactions, daily activities, mobility patterns, physical activity, mood, mental health, wellbeing, nutrition, sleeping patterns, productivity, digitial media use, and psychological situations (Harari et al., 2016; Harari, Müller, \& Gosling, 2020; Mohr et al., 2017; Müller et al., 2020; Rabbi et al., 2011; Sandstrom et al., 2017; Wang et al., 2018; for detailed overviews, see Harari, Müller, et al., 2017, and Vaid \& Harari, 2019). For example, sleep duration can be reliably predicted from phone recharching events, periods when the phone is stationary, and ambient silence (Lane et al., 2011). MSMs are of particular value for personality science, which involves the study of individual differences in human experiences and behavior. For example, mobile sensing data have been used to make inferences about users' personality traits (Stachl, Au, et al., 2020) or to study stable behavioral tendencies in daily life (e.g., Harari, Müller, Stachl, et al., 2020). However, most personality research using MSMs is conducted with Western, Educated, Industrialized, Rich, and Democratic (WEIRD) samples (e.g., Henrich et al., 2010a). Thus, little 
is known about how regional and cultural differences in mobile sensing data might influence psychological inferences drawn from diverse samples.

In this chapter, we provide an overview of psychological mobile sensing research using samples from different regions around the world. We also illustrate the conceptual underpinnings of mobile sensing research and discuss different biases that may occur when MSMs are used in cross-cultural research. We conclude with suggestions on how to mitigate these biases and promote rigorous, fair, and inclusive research in and applications of psychological mobile sensing. We note that there is also a large body of research focusing on the usage of smartphone applications for questionnaire-based ecological momentary assessment, such as experience sampling methods (e.g., Killingsworth \& Gilbert, 2010; Ram et al., 2014; Stieger et al., 2015; von Stumm, 2018; Wahl et al., 2017). However, this chapter solely focuses on research using passive sensing methods.

\section{The State of Cross-Cultural Mobile Sensing Research}

To date, few studies have explicitly looked at how psychological mobile sensing research results differ between cultures or regions. Most psychological studies have focused on mobile sensing data based on one or two countries (Khjawa et al., 2019) or larger samples where regional differences were not accounted for or reported. Furthermore, most mobile sensing research is based on WEIRD samples. Figure 1 maps the locations of smartphone-based passive sensing studies included in the reviews on health and well-being from Cornet and Holden (2018) and on well-being from de Vries et al. (2021). An overview of the included studies and samples can be found in Table 1, and a detailed list can be found on this project's OSF page (https://osf.io/2wbda/). To the best of our knowledge, these are the only existing comprehensive reviews focusing on psychological applications of MSMs. The figure shows that most sensing 
studies were conducted in WEIRD countries and that those studies tended to have greater sample sizes.

\section{Figure 1}

\section{Mobile Sensing Study Locations Around the World}

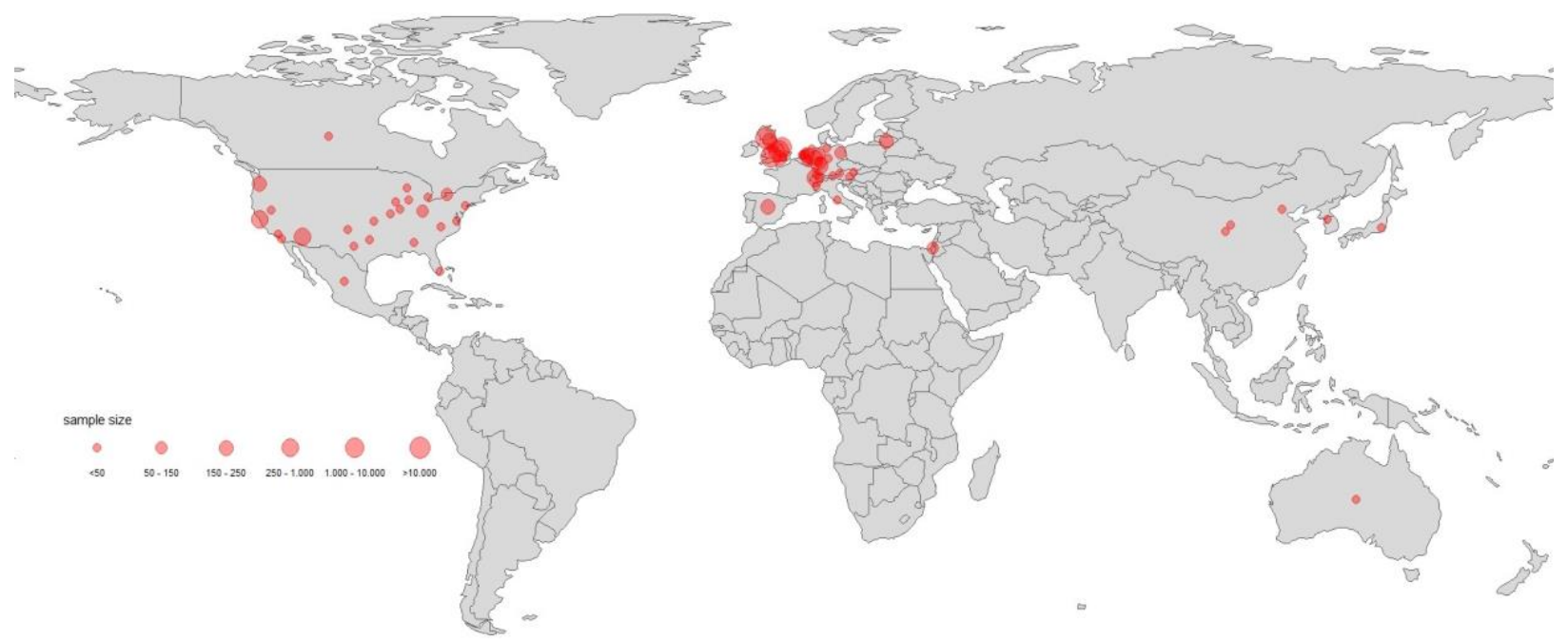

Note. World map depicting the locations and sample sizes of $k=60$ passive sensing studies listed in two reviews (Cornet \& Holden, 2018; de Vries et al., 2021). Studies that did not use passive sensing $(k=27)$ were excluded. Another study was removed as it provided no information on sample size. Provided information on study location was verified using the original papers. For multi-country studies that did not report the sample sizes per country, the total sample size was split evenly across the countries. This figure was created using R (R Core Team, 2014) and the tmap package (Tennekes, 2018). The data file containing a detailed description of all included studies alongside the code that was used to create this figure can be found on this project's OSF page (https://osf.io/2wbda/).

It has yet to be determined to what extent MSM research findings can be expected to generalize across cultures and whether assessments based on MSMs are suitable for comparative research. Cross-cultural generalizability concerns the applicability of research findings to cultural groups other than those under investigation or even to the entire human population (e.g., Deffner et al., 2021; Henrich et al., 2010b; Rad et al., 2018). Future research will show how 
MSMs compare to traditional psychological research tools (e.g., experiments or questionnaires) when it comes to producing cross-culturally robust results that illuminate human universals. However, psychological research is not just concerned with universal phenomena but also with cross-cultural variation in psychological functioning. Comparative research requires equivalent or unbiased assessments, meaning that the same constructs can be measured in the same way in each group (e.g., He \& van de Vijver, 2012; van de Vijver \& Tanzer, 2004). This is a prerequisite for valid interpretations of observed similarities or differences between groups (i.e., cultures; but also groups defined by other variables, such as age, gender, sexuality, disability, ethnicity, or confession). Later in this chapter (see Measurement Bias in Cross-Cultural Mobile Sensing Research), we discuss potential sources of bias in the cross-cultural application of MSMs. Importantly, such biases would not only limit the scope of insights we could gain from MSM research but could also amplify systematic discrimination and social inequalities. For example, MSMs could be used by healthcare providers to determine individualized rates and coverages of health care plans based on outcomes predicted from users' physical activity patterns. However, there might be systematic differences in MSM-based assessments between, for example, income groups that do not reflect actual group differences in physical activity but are due to unequal access to good network coverage and high-quality devices. Such systematic biases in data can then transfer to biases in behavioral inference and algorithmic prediction, thus leading to unfair treatment and systemic discrimination. 


\section{Table 1}

Mobile Sensing Studies and Samples by Continent

\begin{tabular}{|c|c|c|c|c|c|c|}
\hline \multirow[t]{2}{*}{ Continent } & \multirow{2}{*}{$\begin{array}{c}\text { Number of } \\
\text { Studies }\end{array}$} & \multicolumn{5}{|c|}{ Sample Size } \\
\hline & & Total & Range & $M$ & $S D$ & $M d n$ \\
\hline $\begin{array}{l}\text { North } \\
\text { America }^{1,2}\end{array}$ & 25 & 1,117 & $7-229$ & 44.96 & 64.16 & 18 \\
\hline $\begin{array}{l}\text { South } \\
\text { America }\end{array}$ & 0 & - & - & - & - & - \\
\hline Europe $^{2,3,4}$ & 32 & 78,712 & $5-26,700$ & 2461.09 & 6730.77 & 22 \\
\hline Africa & 0 & - & - & - & - & - \\
\hline Asia $^{1}$ & 6 & 165 & $14-91$ & 29.83 & 30.60 & 15 \\
\hline Oceania $^{3}$ & 1 & 43 & - & 43 & - & 43 \\
\hline
\end{tabular}

Note. Overview of the $k=60$ passive sensing studies used to create Figure 1 grouped by continent. Multi-country studies (see below) were included once per continent. Therefore the sum of the number of studies adds up to 64 . ${ }^{1234}$ For details on the multi-country studies, see the list of included studies available on this project's OSF page at https://osf.io/2wbda/.

To the best of our knowledge, only one study from Khwaja et al. (2019) has explicitly considered the cultural context in mobile sensing research. In this study, the authors compared the performance of supervised machine learning (ML) models predicting Big Five personality traits from mobile sensing data collected from 545 participants across five countries (UK, Spain, Columbia, Peru, and Chile). To test whether algorithms trained on culturally diverse samples generalize to new countries, the authors applied a leave-one-country-out cross-validation technique. In this approach, the algorithm was trained using data from four countries, and the algorithm was then tested on data from the excluded country. This procedure was repeated five times, excluding a different country in each iteration. Additionally, the authors examined how well culture-specific models performed (i.e., training and testing a model with data from the 
same country). Their results indicated that using data from the same country to train and test the models (i.e., culture-specific models) significantly improved prediction accuracies for extraversion, agreeableness, and conscientiousness, while neuroticism and openness seemed to be culture-robust (i.e., the results were comparable for both approaches). Müller et al. (2021) used a similar approach to investigate whether depression can be accurately predicted from GPS data in a diverse US-based sample and in socio-demographically homogeneous sub-samples. In this study, one algorithm was trained on the entire sample and then tested in homogeneous groups (e.g., women living in rural areas, or highly educated men), while another set of algorithms was trained and tested within each sub-sample. However, the predictive accuracies achieved in a student sample - a highly specific subculture - could not be replicated in other groups, further underscoring the need for generalizability research.

In addition to a lack of comparative mobile sensing research, few studies have utilized non-WEIRD samples. Moreover, the majority of the non-WEIRD studies we reviewed do not explicitly report sample demographics, meaning that the cultural composition of the samples could only be speculated on based on the location of the authors, description of the data collection process, and the language of the survey instruments used. For example, three studies likely used Chinese samples to examine the prediction of mood (Ma et al., 2012), emotional states (Zhang et al., 2018), and sleep quality (Bai et al., 2012) from smartphone data, but do not explicitly state so. Another recent study used a sample of Chinese university students to associate the Big Five personality traits with sensor-based daily spatial behavior obtained from telecommunication companies (Ai et al., 2019). Lee et al. (2014) likely used a Korean sample to examine correlations between smartphone addiction and daily smartphone use frequency and 
duration. Lastly, one study predicted loneliness from smartphone data in a sample of older adults likely based in Mexico (Sanchez et al., 2015).

To sum up, there are relatively few empirical findings that can serve as a basis to evaluate the generalizability of psychological mobile sensing research across cultural contexts (especially between WEIRD and non-WEIRD samples). Therefore, the following sections will illustrate factors that might threaten cross-cultural MSM research from a conceptual perspective. To this end, we first outline the underpinnings of MSM research. We then elaborate on potential biases that can occur in cross-cultural MSM research and conclude this chapter with strategies for bias mitigation. We note that most of the following considerations do not only apply to comparisons between cultures but also between groups defined by other variables (e.g., gender, age, etc.).

\section{Underpinnings of Mobile Sensing Research}

To illustrate the types of biases that may occur in mobile sensing data and jeopardize cross-cultural comparisons, we first must understand the conceptual underpinnings of mobile sensing research. We will focus on MSM applications for behavioral assessment (i.e., measurement of person variables; e.g., Harari et al., 2016; Harari, Müller, et al., 2017) rather than situational assessment (i.e., measurement of environment variables; e.g., Harari, Müller, \& Gosling, 2020), though these are often closely linked or overlapping. For example, GPS data can be used to examine mobility behaviors or locations, and microphone data can indicate verbal behavior or social situations. Sensors are not designed to capture behaviors per se but rather to measure changes in the physical or chemical properties in their surroundings (Yan \& Chakraborty, 2014). Behavioral sensing relies on the assumption that these changes are, at least on average, due to the behavior of the person in possession of the sensing device (i.e., the user); therefore, sensor data will contain rich behavioral information about a user. For example, an 
accelerometer measures the acceleration of a device. If a user carries the device, accelerometer data can then be used to infer whether they are walking, running, or stationary (Harari, Gosling, et al., 2017). Furthermore, unobserved behavior can be inferred from behavioral products, that is, the traces people's behaviors leave behind in their physical or digital environments (i.e., indirect measurement of person variables via measurement of environment variables). For example, light sensors measure the illuminance of surroundings but can be used to infer that a person has stepped outside or switched off the lights (together with other information). However, sensors can only capture behaviors or behavioral products associated with the physical properties they are designed to measure. For example, accelerometers are suitable for sensing physical movement, GPS data can record mobility patterns, microphones can capture social interactions, and light sensors can be used to infer sleep patterns (Harari et al., 2016; Harari, Müller, et al., 2017; Schoedel et al., 2020). This means that the availability of different types of sensors restricts the range of behaviors that can be captured.

In addition to actual sensors, metadata logs are commonly used in MSM research (Harari et al., 2016; Harari, Müller, et al., 2017). For example, phone logs record occurrences in software runs that can capture mediated behaviors (i.e., behaviors carried out through the device) such as smartphone-mediated communication (e.g., frequency and duration of calls or number of unique contacts texted) or other mediated activities (e.g., duration and frequency of phone or app usage; Harari, Müller, et al., 2017). However, logs only contain data about data (i.e., metadata) and may often have limited psychological relevance. For example, log data does not specify whether an outgoing call was made to chat with a friend, to order a pizza, or to report an issue to customer service. Furthermore, the extent to which a person's phone logs are representative of their 
computer-mediated behaviors depends on whether they use other devices for similar purposes (e.g., make calls from a landline or use messenger apps on a tablet or PC).

Importantly, MSM-based behavioral assessment is always indirect because some degree of inference is necessary to link the sensor or log data to actual behavior. Therefore, the target behavior should be considered a latent variable reflected in the data and inferred from indicators (i.e., features) through a specific computational process. For some behaviors, this can be done automatically using software already integrated into the device. For example, smartphone pedometers infer the number of steps a person has taken from data generated by sensors (mostly accelerometers, gyroscopes, and digital compasses). Certain mediated behaviors can be inferred from metadata logs with ease and near-perfect accuracy. For example, phone log data can be used to infer when a person has made a phone call. Other behavioral inferences require more complex computational processes to extract meaningful quantitative variables or features. This is often done using classifiers such as ML algorithms designed to compute relevant variables. For example, audio classifiers have been used to infer conversation behavior from microphone data (e.g., Harari, Müller, et al., 2017). By combining data captured by multiple sensors, more specified or contextualized behaviors can be inferred (Harari, 2016). Wang et al. (2014) used combined data from location sensors, microphones, and motion sensors to infer studying behavior of college students. Behavioral assessments are often aggregated across occasions or combined with other behavioral variables to create new variables representing individual behavioral tendencies or composite constructs. Harari, Müller, Stachl, et al. (2020) estimated individual tendencies for several communication behaviors by computing within-person averages across different time periods and using principal component analysis to create formative composite variables for texting or calling behavior. 
Behavioral variables assessed using MSMs can serve as indicators of broader latent psychological constructs (Harari et al., 2021), such as momentary states (e.g., personality states, emotions, and momentary well-being) or stable traits (e.g., Big Five personality, cognitive abilities, and general well-being). Stachl, Au, et al. (2020) used ML techniques to predict Big Five personality domains and facets from a variety of behavioral variables inferred from smartphone data. To be inferable from mobile sensing data, a target construct needs to manifest in tangible behavioral cues that are machine-readable (i.e., that can be captured by sensors or metadata logs). As a result, target construct and behavioral cues covary, enabling the prediction of the construct from mobile sensing data. The externalization process (target construct(s) $\rightarrow$ behavioral cue) can be depicted using a Brunswikian lens model (see Figure 2; Phan \& Rauthmann, 2021; Vinciarelli \& Mohammadi, 2014). In a Brunswikian framework, the informativeness of a cue for inferences about the target construct is called its ecological validity (Brunswik, 1956). However, as behavior is contextualized and determined by both the person and the situation (Rauthmann, 2016, 2021), ecological validity is not an inherent property of the cue but can vary by person, situation, and time. For example, "dancing wildly" may be an indicator of state extraversion but may be indicative of conscientiousness when executed by a professional dancer during an audition (Horstmann \& Ziegler, 2020). We argue that different behaviors can also differ in how indicative of a target construct they are across different cultural contexts. 
Figure 2

Brunswik Lens Model Framework Illustrating the Underpinnings of Mobile Sensing

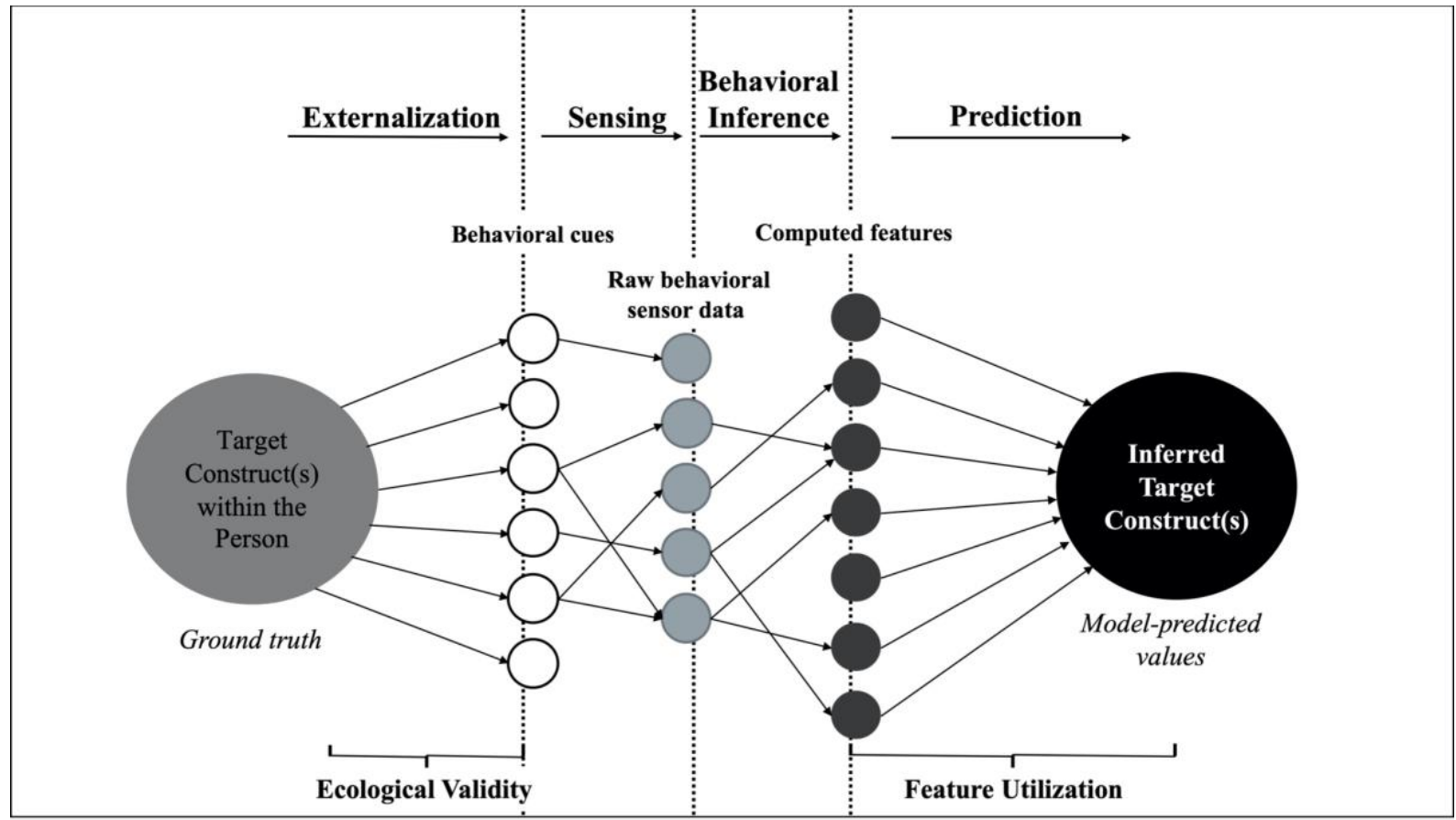

Note. Brunswikian lens model depicting the underpinnings of psychological mobile sensing. Behavioral cues relevant to one or multiple target constructs are externalized by a person. These cues are partly captured by mobile sensors. The raw sensor data is then used to compute features (i.e., behavioral variables) which, in turn, can be used to infer the target construct(s) using traditional statistical techniques or machine learning.

Next, behavioral cues are captured by different sensors or manifest in data logs (behavioral cues $\rightarrow$ raw behavioral sensor data; see Figure 2). Depending on both the nature of the target construct and the capabilities of the device, not all relevant behavioral cues externalized by a person may be machine-readable. Furthermore, several factors may affect the reliability and validity of behavioral inferences from sensor data, including the properties of the specific sensing device, how the device is being used, and the environment of the user (see Measurement Bias in Cross-Cultural Mobile Sensing Research). Assuming sensors and metadata 
logs have at least partially captured behavioral cues that are informative of the target construct, we now have raw sensor data that usually requires preprocessing (i.e., similar to data cleaning) and subsequent computation of features (sensor data $\rightarrow$ computed features; see Figure 2 ) for further analysis. When the goal is to infer or predict a stable construct (e.g., a personality trait), features are usually computed as behavioral summary statistics (e.g., measures of central tendencies or variability) across certain time windows (e.g., daily averages). Extracted features can then be used for traditional statistical analyses (e.g., Wang et al., 2018), or they can serve as input data for ML models. ${ }^{1}$ Psychological mobile sensing research commonly uses supervised ML, which concerns the automatic prediction of known values of the target construct (i.e., the "ground truth" assessed using some standard criterion, e.g., self-report questionnaires) from the input data (Stachl, Pargent, et al., 2020; Tay et al., 2021). The ML algorithm selects and weights features based on training data to optimize a function that best predicts the ground truth (Lantz, 2019). The performance of the algorithm (i.e., predictive accuracy) is then evaluated using test data (known input and output; not used in training). ${ }^{2}$ If the testing yielded good results, the ML algorithm could then be applied to new data (i.e., known input data but unknown output).

In the next section, we elaborate on different types of measurement bias sources and implications for cross-cultural mobile sensing research. We focus on MSMs in the application of latent construct assessment, which may be particularly susceptible to biases since systematic errors can be introduced at each step (i.e., externalization, sensing, feature extraction, and, when

\footnotetext{
${ }^{1}$ There are studies that do not aim to predict a latent target variable or other outcome variables from mobile sensing data (and inferred behaviors but solely examine manifest behavioral patterns. Such accounts are important to describe inter- and intra-individual patterns of behavioral expression (including change and stability) and can also inform theory (i.e., explanatory account; Harari, Vaid, et al., 2020).

${ }^{2}$ Very high predictive accuracies are unlikely and may be due to overfitting issues because features and ground truth come from different data sources and methods. Predictive accuracy in ML, like convergent validity in traditional psychometric assessment, is limited due to method artifacts and unique information in different data sources. It is also attenuated by measurement error and poor construct coverage. For predictive accuracies in personality computing, Wiernik et al. (2020) estimated ceilings to convergent validity at $r \approx .30-.50$.
} 
applicable, prediction). Furthermore, due to the hierarchical structure of the lens model, these biases can have cascading effects: Biases in earlier steps (e.g., externalization) will affect the data generated at later steps (e.g., feature extraction). We restrict our examination of potential biases in mobile sensing to those that may occur when ML is not used (or before ML is used) to infer the target construct. Biases specific to ML applications lie outside the scope of this chapter, and readers are referred to Tay et al. (2021) for an examination of measurement bias in MLbased psychometric assessment.

\section{Measurement Bias in Cross-Cultural Mobile Sensing Research}

Measurement bias occurs when differences in observed variables cannot be fully explained by true differences in what is intended to be measured (e.g., He \& van de Vijver, 2012; Oort et al., 2009; van de Vijver \& Tanzer, 2004). Formally, this is a violation of measurement invariance or measurement equivalence. Measurement invariance means that the function that relates observed indicators to the latent target variable or measurement model is the same across groups (Borsboom et al., 2008; Oort et al., 2009; Putnick \& Bornstein, 2016). ${ }^{3}$ Measurement models are commonly specified using confirmatory factor analysis (CFA) or item response theory. Novel big data approaches, including many sensing studies, often use ML, which can be considered a special type of measurement model (Tay et al., 2021). Independent of the measurement model, measurement bias implies that some portion of observed variance cannot be attributed to the target construct or to random measurement error and is instead due to systematic error (i.e., nuisance factors; He \& van de Vijver, 2012; van de Vijver \& Tanzer, 2004). When a nuisance factor varies systematically across cultural samples, observed cross-cultural differences

\footnotetext{
${ }^{3}$ Measurement invariance is typically discussed with regard to personality traits, cognitive abilities, or other broad psychological constructs. However, it is also a concern when the latent target variable is a behavior (e.g., an activity) that is not directly observed but inferred from manifest indicators or when the target construct is a formative composite variable (e.g., a behavioral tendency).
} 
may not reflect true differences in the target construct. Therefore, "insight into the presence and strength of biasing effects is a prerequisite for sensible group comparisons to be made" (Borsboom, 2006, p. 178). While this chapter focuses on cross-cultural research, it is noteworthy that measurement bias may, of course, also exist regarding other socio-demographic or grouptype variables (e.g., gender, age group, or education level) or dimensional attributes (e.g., latent traits or abilities; Meredith, 1993; Oort et al., 2009) that may vary within cultures.

He and van de Vijver (2012; see also van de Vijver \& Tanzer, 2004) distinguished different types of biases in cross-cultural research based on the location of the systematic error source (i.e., construct level, the method level, or item level). Though this taxonomy was proposed for traditional assessment procedures (e.g., cognitive tests, questionnaires, or behavioral observation; van de Vijver \& Tanzer, 2004), similar biases can be identified in crosscultural mobile sensing research. Furthermore, these biases can occur in sensing research when passive sensing is combined with experience sampling methods or other questionnaire measures, for example, to assess the ground truth for supervised ML (Tay et al., 2021).

First, construct bias occurs when the definition of the target construct differs across cultures or "when not all relevant behaviors associated with the construct are present and properly sampled in each culture" (He \& van de Vijver, 2012, p. 5). We argue that mobile sensing can be susceptible to this type of bias when a target construct is differentially externalized across cultures or when cross-cultural differences exist in the functional implications of relevant behaviors.

Second, method bias is a broad category of systematic errors rooted in research methods (He \& van de Vijver, 2012; van de Vijver \& Tanzer, 2004) and can be further distinguished into more specific types of bias. Sample bias concerns the representativeness and comparability of 
cultural samples. In particular, recruitment procedures and technical requirements may influence the composition of mobile sensing study samples. In the sensing process (see Figure 2), systematic errors may be introduced by devices and the behavior of participants (Blunck et al., 2013). Device-type bias results from systematic differences in the devices used by individuals in different cultural samples. Device properties and capabilities determine the types of sensing data they can provide as well as the quality of the data.

Third, bias from user practices can occur in cross-cultural MSM research when participants from different cultural samples vary in how they use and customize their devices. This can have a bearing on both the quantity and quality of recorded sensing data. While potential biases from device properties and user practices uniquely concern MSM-based assessment, many other biases that can occur in the application of traditional psychometric instruments do not affect MSMs. These include biases due to systematic cross-cultural differences in (a) response styles (e.g., acquiescence, extreme responding, socially desirable responding); (b) familiarity with test properties (e.g., the test/response format or the stimulus material), or (c) responses to test administration conditions (e.g., physical and social conditions, instructions, or behavior of the tester; He \& van de Vijver, 2012; van de Vijver \& Tanzer, 2004). Lastly, item bias (or differential item functioning; see, e.g., Bauer, 2017; Borsboom, 2006) occurs when an item score covaries with cultural group membership. This can be the case when an item has been poorly translated or adapted to different cultural contexts (He \& van de Vijver, 2012; van de Vijver \& Tanzer, 2004). Since mobile sensing does not use items in a psychometric sense, inappropriate instrument translation and adaptation is only a concern when passive sensing is combined with traditional assessment methods (e.g., self-reports). The smallest meaningful quantitative unit in mobile sensing is a single feature extracted from the raw 
data. Akin to item bias, a feature can be nonequivalent across groups, for example, when it has been computed from speech or written texts in different languages (Tay et al., 2021).

In the following sections, we consider potential sources of bias in psychological MSM research (see Table 2 for an overview). We will discuss biases that may be caused by crosscultural differences in (a) the target construct and its behavioral manifestations; (b) sample characteristics; (c) device properties; and (d) user practices. Furthermore, we will illustrate how (e) environmental factors may systematically affect sensing data and sensor-based behavioral inferences. We focus on bias that may occur at the construct level, in behavioral expressions, or during the sensing process (see left half of Figure 2). Computational biases are outside the scope of this chapter, but interested readers may refer to Tay et al. (2021) for an examination of measurement bias in ML-based psychometric assessment. 
Table 2

\section{Potential Biases in Cross-Cultural Mobile Sensing Research}

\begin{tabular}{|c|c|c|c|}
\hline $\begin{array}{c}\text { Biases } \\
\text { rooted in }\end{array}$ & Description and causes & $\begin{array}{c}\text { Potential nuisance factors } \\
\text { (some examples) }\end{array}$ & Illustrative example \\
\hline $\begin{array}{l}\text { Target } \\
\text { construct and } \\
\text { its behavioral } \\
\text { manifestations }\end{array}$ & $\begin{array}{l}\text { People from different cultures express different behaviors for the same } \\
\text { level of the target construct. Behavioral manifestations of the target } \\
\text { construct in each culture depend on cultural norms and environmental } \\
\text { factors (e.g., opportunities and constraints). }\end{array}$ & $\begin{array}{l}\text { - social appropriateness or legality } \\
\text { of certain behaviors } \\
\text { - function and cultural meaning of } \\
\text { certain behaviors }\end{array}$ & $\begin{array}{l}\text { In Country A, sidewalks are everywhere and } \\
\text { most people walk to get from one location to } \\
\text { another. In Country B, sidewalks are rare and } \\
\text { when people walk, they do so mostly for } \\
\text { exercise. }\end{array}$ \\
\hline $\begin{array}{l}\text { Sample } \\
\text { characteristics }\end{array}$ & $\begin{array}{l}\text { Cultural samples are not representative or not comparable. Sample } \\
\text { compositions depend on which subpopulations were targeted by } \\
\text { recruitment procedures and selected due to study requirements (e.g., } \\
\text { device ownership, device-type requirements) in each culture. }\end{array}$ & $\begin{array}{l}\text { - demographic variables (e.g., age, } \\
\text { gender, education) } \\
\text { - psychological variables (e.g., } \\
\text { motivation, traits) }\end{array}$ & $\begin{array}{l}\text { A freely available smartphone sensing app for } \\
\text { Android is released. In Country A, most people } \\
\text { use Android phones. In Country B, Android } \\
\text { users tend to be older males with lower incomes. }\end{array}$ \\
\hline $\begin{array}{l}\text { Device } \\
\text { properties }\end{array}$ & $\begin{array}{l}\text { Relevant properties of software platforms or hardware vary } \\
\text { systematically across cultural samples. Different devices may be used } \\
\text { by participants from each cultural sample, e.g., due to regional } \\
\text { differences in platform or device model market shares. Ambient factors } \\
\text { that vary systematically across cultural regions (e.g., climate, } \\
\text { geography) can also affect device performance (e.g., sensor accuracy). }\end{array}$ & $\begin{array}{l}\text { - platform policies } \\
\text { - processing power } \\
\text { - sensor types } \\
\text { - sensor performance }\end{array}$ & $\begin{array}{l}\text { Most participants from Country A have an X- } \\
\text { phone which tends to undercount steps. Most } \\
\text { participants from Country B have a Y-phone } \\
\text { which tends to overcount steps. }\end{array}$ \\
\hline User practices & $\begin{array}{l}\text { Participants from different cultural samples vary systematically in how } \\
\text { they handle and use their devices. Cross-cultural variation in user } \\
\text { practices may be due to differences in cultural norms or environmental } \\
\text { factors (e.g., device-related infrastructure). }\end{array}$ & $\begin{array}{l}\text { - device carrying behavior (when, } \\
\text { where, and how) } \\
\text { - (re)charging behavior } \\
\text { - device sharing } \\
\text { - customized device settings }\end{array}$ & $\begin{array}{l}\text { Participants from Country A tend to carry their } \\
\text { smartphones in their pockets which allows for } \\
\text { fairly accurate step counts. Participants from } \\
\text { Country B tend to carry (and leave) their phones } \\
\text { in backpacks or handbags which leads to steps } \\
\text { being undercounted. }\end{array}$ \\
\hline $\begin{array}{l}\text { Environmental } \\
\text { factors }\end{array}$ & $\begin{array}{l}\text { Cultural samples live in different environments. External factors may } \\
\text { systematically affect mobile sensing data in several ways, such as } \\
\text { (a) by influencing participants' behavior (i.e., behavioral } \\
\text { manifestations or user practices; see above); } \\
\text { (b) by impairing device performance (see above) or sensing app } \\
\text { functionality (e.g., due to poor network coverage); } \\
\text { (c) by introducing non-behavioral variance (e.g., background noise in } \\
\text { audio recordings); } \\
\text { (d) by dictating how data can be collected and stored (i.e., local laws). }\end{array}$ & $\begin{array}{l}\text { - opportunities and constraints } \\
\text { afforded by the environment } \\
\text { - ambient factors (e.g., climate, } \\
\text { geography, noise) } \\
\text { - device-related infrastructure } \\
\text { (e.g., opportunities for charging, } \\
\text { network coverage, data cost) } \\
\text { - legal frameworks (e.g., data } \\
\text { protection and privacy laws) }\end{array}$ & $\begin{array}{l}\text { In County A, mobile data is rather inexpensive } \\
\text { and charging stations are widely available (e.g., } \\
\text { at bus stops and at restaurants). In Country B, } \\
\text { mobile data is expensive and there are few } \\
\text { opportunities for charging. Many people } \\
\text { routinely disable mobile data to save money and } \\
\text { battery power. }\end{array}$ \\
\hline
\end{tabular}

Note. Bias occurs in cross-cultural data when observed differences between cultural samples do not fully correspond to true differences in the target construct.

This is the case when some nuisance factor that affects the data varies systematically across cultural samples. 


\section{Construct Bias and Nonequivalent Behaviors}

A person's behavior depends not only on their stable traits and their current states but also on the situation and the broader environmental context in which it occurs (Rauthmann, 2021). Across cultures and regions, behavior is influenced by different presses, affordances, demands, and resources provided by the respective socio-cultural and/or physical environments. Specific behaviors may be unique to certain groups (e.g., rituals) or are only possible in certain locations (e.g., taking the subway). For behaviors that can be observed across cultures, the psychological meaning may not be equivalent for several reasons. First, cross-cultural differences may exist in the normativeness or appropriateness of certain behaviors (which may additionally be age-graded or sex-dependent so that other third variables also need to be consulted). For example, littering is socially undesirable in most places, but it can be a serious violation of both cultural norms and strictly enforced local laws in some regions (van de Vijver \& Tanzer, 2004). Second, the same behavior can serve different functions in different cultures. For example, in some cultures, people may mostly ride bicycles for exercise, while in other cultures, people cycle to get from A to B. Third, different behaviors can serve the same functional purpose. For example, people from some cultures may exercise by cycling or running, while people from other cultures go to the gym. Whether and why people cycle may depend on socio-cultural norms but also on other environmental factors, such as the availability and affordability of other modes of transport (e.g., cars or trains) and of other means of exercise (e.g., gyms), the climate, or road safety. Similarly, how people use their smartphones (i.e., devicemediated behaviors) will vary across cultures depending on norms, but also on, for example, the availability of functionally similar alternatives (e.g., whether people also own a PC or tablet), phone plan subscriptions, and network coverage. 
As a result, people from different cultures may express different behaviors reflecting the same level of the latent construct (He \& van de Vijver, 2012; van de Vijver \& Tanzer, 2004, Tay et al., 2020). In the lens model framework (see Figure 2), this means that a behavioral cue may possess high ecological validity (i.e., be a valid indicator of the target construct) in some cultures but not in others. Importantly, cross-cultural differences may not only exist in how a construct is externalized but also in the extent to which it is externalized at all. Via sensors or metadata logs, MSMs can only directly capture overt behaviors expressed in the physical or digital space using sensors and logs, respectively - but not covert intra-psychological (e.g., perceptual, cognitive, affective, or motivational) states. Assessing the latter would require additional data sources (e.g., self-reports) or analysis (e.g., ML algorithms trained to infer mental states from behavioral data). Therefore, MSMs may be particularly susceptible to construct bias when the extent to which the target construct is externalized (i.e., expressed in observable or machinereadable behavior) varies between cultures. This may be the case, for example, when cultural norms encourage or discourage the behavioral externalization of socially evaluative traits (e.g., intelligence or ignorance; John \& Robins, 1993), or when the target construct or associated behaviors are differentially stigmatized across cultures (e.g., depression; Krendl \& Pescosolido, 2020). Furthermore, not all relevant behavioral cues may be appropriately sampled (or sensed) in each culture, for example, because they were identified based on previous research or by researchers from one culture. This would not only be problematic in latent construct assessment but also when determining the behaviors that constitute a formative construct. For example, typical means of smartphone-mediated communication identified in one culture (e.g., calling, texting, and certain messaging apps) may not include all relevant communication channels commonly used in another culture (e.g., other apps or browser-based clients). 


\section{Sample Bias}

Cross-cultural comparison is valid only if the samples are representative of the target population within a given culture (e.g., Deffner et al., 2021) and comparable between cultures (e.g., He \& van de Vijver, 2012). Study participant selection is based on several factors, such as (a) the reach of the recruitment campaign; (b) how the study is advertised and what incentives are offered; and (c) eligibility criteria. These factors establish a bottleneck that may inadvertently introduce sample bias. For example, when a study aims to recruit large samples from the general population by releasing and promoting a freely available smartphone application, different demographics will be targeted depending on how the app is advertised, what it promises, and what it requires. In cross-cultural research, the bottleneck created by recruitment efforts and study requirements may produce different sample compositions in different cultures. For example, participation in smartphone sensing studies requires owning a smartphone and, while smartphone usage continues to increase worldwide, there is still considerable cross-national variation. According to a 2018 survey from Pew Research Center (Silver, 2019), 95\% of adult South Koreans owned a smartphone, and smartphone ownership was very common across age groups, genders, or education levels. In India, on the other hand, only $24 \%$ had a smartphone, and these were mostly young men who had completed secondary education. Furthermore, participants' devices need to meet the technical requirements of the sensing app, which means that only certain user groups (e.g., only Android users) are eligible to participate. This can lead to biased samples when the sociodemographic characteristics of users differ systematically across phone models or platforms (e.g., CivicScience; Götz et al., 2017). As device-type demographics (Blunck et al., 2013) likely also vary between cultures, the range of supported devices (e.g., Android only) can result in different sample compositions. Importantly, even seemingly 
matching samples (e.g., college students) may vary substantially in their demographics or in their motivation, depending on the culture they come from and how they have been recruited (He \& van de Vijver, 2012; van de Vijver \& Tanzer, 2004).

\section{Device Type Bias}

When MSM study participants use their own device (rather than identical, calibrated ones handed out by the researchers) - as is the case in many smartphone sensing studies - their devices are also sampled. This means that assessments lack full standardization because different measurement tools (i.e., devices) are used to collect data from different people. The unique properties of different devices can have a bearing on the data and thus become potential sources of error at the person level. These aggregate to group-level biases when the distribution of different smartphone manufacturers and models varies systematically between cultural samples. This may be the case, for example, when samples are recruited from countries or regions with different phone model market shares (e.g., Counterpoint, 2021) or when device-type demographics vary across cultures (see Sample Bias). The devices of people sampled from different cultures may not be equipped with the same software and hardware and therefore may not provide sensing data of equal quality. Device heterogeneities exist across (a) platforms (e.g., in distribution and app store policies or in the specifications of different application programming interfaces); (b) smartphone hardware (e.g., in sensor performance, sampling rate, or processing power); and (c) software and hardware version updates (Blunck et al., 2013). Here, we focus only on biases due to variation in sensing capabilities across different devices, and we refer readers to Bluck et al. (2013) for an overview of other device heterogeneities examined from a computer science perspective. As the availability of different types of sensors dictates the types of behaviors that can be captured, devices equipped with a wider array of sensors might be 
able to access a broader range of behavioral expressions. Devices differ not only in the types of sensors they contain, but also in the performance of those sensors (i.e., their precision and accuracy). Precision can be seen as the psychometric reliability of a sensor. A precise sensor produces data that is mostly free from unsystematic error or noise. Sensor accuracy (i.e., trueness; akin to psychometric validity) refers to the degree to which a sensor is free from systematic error or sensor bias. For example, if a person takes exactly 100 steps at multiple occasions, a precise pedometer will show little variability in step count and the pedometer is accurate if the average indicated step count across occasions is close to 100 . The performance of different sensors varies across different types of devices but also between individual devices of the same model (e.g., Chaffin et al., 2017; Kuhlmann et al., 2021; Stisen et al., 2015; Woo et al. 2020). Smartphone sensors are often poorly built and calibrated (e.g., Blunck et al., 2013; Grammenos et al., 2018; Stisen et al., 2015) because manufacturers want to reduce costs and because sensors only need to perform sufficiently well for their intended uses which do not include scientific behavioral assessment. Sensor accuracy may also change slowly over time (natural drift; e.g., Kuhlmann et al., 2021) or due to external forces, such as when the device is dropped (Stisen et al., 2015). Furthermore, environmental factors that can directly affect sensor performance may vary systematically across cultural regions. For example, motion sensor readings can be skewed in warmer climates (e.g., Kos et al., 2016) and GPS signals can be blocked by buildings or trees (e.g., Ma et al., 2020; Bastos \& Hasegawa, 2013).

\section{Bias from User Practices}

People differ in how they use and handle their smartphones, and these individual differences in user practices are reflected in sensed data (Blunck et al., 2013). When typical user practices vary between cultures, observed cross-cultural differences (or similarities) in sensed 
behaviors must be interpreted with care. As far as we know, there is not a single cross-cultural investigation into different user practices, let alone a study of their implications for MSMs. Still, there is every reason to believe that cross-cultural differences exist. User practices encompass all smartphone-related behaviors that are not device-mediated activities (e.g., app usage) or communication behaviors (e.g., calling or texting). These include, for example, how often and in which contexts people have their smartphones on them, how they carry their phones, how frequently they recharge them, or whether they let other people use their phones. Needless to say, sensors can only capture behaviors that are enacted while the sensing device is on or near a person. For example, if a person leaves their phone at home when they go for a walk in the park or if it runs out of battery, it cannot count any steps. Another person who never leaves the house without their phone and never lets it run out of battery might get a higher step count, even if they have actually taken fewer steps. Furthermore, both the precision (i.e., reliability) and accuracy (i.e., validity) of the step count may not only depend on the performance of the sensors (see Device-Type Bias) but also on how the device is carried. For example, pedometers tend to be most accurate when the phone is carried in a pants pocket (e.g., Åkerberg et al., 2012; Leong \& Wong, 2016), so if the phone is carried in a handbag or in the chest pocket of a jacket, the step count may be off. Across cultures, how people typically carry their phones may depend on what is in fashion (e.g., whether current devices fit into pockets on current pants), what smartphones are typically used for (e.g., whether people need their phones close at hand because they use it to pay for the subway), and others' behavior (e.g., whether phones are frequently stolen). Sensing data are also influenced by how people customize their phone settings and how they use different functions, including the functions of a sensing app used for research. For example, some people may routinely disable certain features (e.g., Bluetooth, GPS, or WiFi) or pause sensing in the app 
to save data and battery power (Blunck et al., 2013). Further, they might be more likely to do so when they live in regions where opportunities for charging or public WiFi hotspots are rare. Cross-cultural differences in user practices can systematically affect (a) how continuously behavioral data can be collected per person and (b) in which contexts or situations data is collected, (c) how noisy (i.e., imprecise/unreliable) the data is, and (d) how accurately (i.e., validly) behavior can be captured.

\section{Bias from Environmental Factors}

Environmental factors are any external factors (e.g., physical, political, legal, economic, or socio-cultural) that may, directly or indirectly, affect the data collected via MSMs. In the previous sections, we have already discussed potential effects of environmental variables on people's behavior, including non-mediated and device-mediated behaviors that may be target variables or indicators in MSM-based assessment (see Nonequivalent Behaviors and Construct Bias) as well as user practices that impact the amount and quality of recorded data (see Bias from User Practices). We have also pointed out that environmental factors can compromise sensor performance (see Device Type Bias). Furthermore, sensors may directly record the environment, and it can be difficult to separate behavioral variance from environmental variance during feature extraction. For example, if we want to infer conversation behavior from microphone sensors, living in crowded and noisy areas can lead to an overestimation of social interactions. Beyond affecting externalization, sensing, and feature computation (see Underpinnings of Mobile Sensing Research, and Figure 2), environmental variables also set the framework under which a sensing study can be conducted. For example, legal aspects dictate how data can be collected, processed, stored, and analyzed. The European Union's General Data Protection Regulation (GDPR) represents one of the world's strictest and most comprehensive data protection laws 
treating personal information protection and privacy as fundamental rights. In contrast, U.S. data privacy laws are limited by the right to freedom of speech following a minimalistic approach. China's legal framework can be located somewhere between the EU and the U.S.'s stance on data protection but also establishes some specificities, such as the principle of cyber-sovereignty (i.e., application of state sovereignty to cyberspace; for a detailed comparison, see PernotLeplay, 2020). National differences in data protection and privacy laws systematically affect the data collection process and the data itself (e.g., What type of data can be collected? How and where should data be stored?). Stricter data protection laws ensure participants' privacy, but they also make it more difficult to collect psychologically rich data containing information about why and how people show specific behaviors. For example, the content of a person's text conversations contains more psychologically meaningful information than the metadata text logs alone. Another environmental factor affecting smartphone sensing studies is mobile network coverage which can differ widely between countries (Delaporte et al., 2021). Poor network coverage can affect data quality and may result in a higher proportion of excluded data or invalid behavioral inferences.

\section{Bias Mitigation Strategies for Mobile Sensing Research}

In the previous section, we outlined possible biases in mobile sensing research and their sources (see also Table 2 for an overview). When data is not collected under strictly controlled laboratory conditions, but instead in people's everyday lives, it is nearly impossible to control for all potential error sources. However, there may be ways to prevent, minimize, or measure and statistically account for the influence of nuisance factors. In this section, we propose mitigation strategies that could be applied to reduce biases and promote fair and methodologically sound approaches to cross-cultural mobile sensing research (see Figure 3 for an overview). We go 
through the broad stages of a generic mobile sensing study and point out mitigation strategies to consider at each stage. The strategies presented here are not exhaustive and may not be applicable to every research endeavor. Furthermore, individual projects might afford completely different approaches tailored to their specific research questions, target variables, samples, or methods.

\section{Figure 3}

Possible Bias Mitigation Strategies During the Planning and Implementation of a CrossCultural Mobile Sensing Study

\section{Study planning}

O Ensure construct equivalence by defining target constructs explicitly

O Formulate hypotheses about potential cross-cultural differences in nomological networks O Ensure sufficient knowledge about target constructs as well as cultural and environmental factors that might affect their manifestations (e.g., with the help of informants)

O Operationalize constructs to be comparable across cultures (e.g., with the help of pilot studies, decentering, and convergence)

O Define a target population that exists in each culture and is comparable across cultures O Capture relevant individual and demographic variables and statistically investigate differences across cultures

\section{Study implementation}

O Ensure inclusive recruiting of representative samples via culture-specific recruiting strategies and incentives (e.g., developed with the help of informants)

O Properly translate and adapt study information and instructions

O Train investigators and research assistants in intercultural communication

O Aim for consistent user practices across cultures by providing clear instructions and incentives

O Capture and statistically control for individual differences in user practices

O Use standardized or calibrated devices if possible, or limit functionality and sensing to what the most restrictive platforms and devices allow

In the planning stage, the research question is formulated, and a study is designed to address this question. First, researchers need to define the target quantity and target population and think about how each can be empirically approximated in each culture (Deffner et al., 2021; 
Lundberg et al., 2021). ${ }^{4}$ To ensure construct equivalence, psychological constructs should be explicitly defined, and testable statements should be formulated about potential cross-cultural differences in nomological networks (van de Vijver \& Tanzer, 2004). This requires a sufficient knowledge of not only the construct itself but also cultural and environmental factors that might affect associated behavioral manifestations. For formative behavioral constructs (e.g., exercising), equivalence can be accomplished by carefully considering which behaviors may constitute the construct in each culture. Informants can provide expertise in local culture and regional distinctions (He \& van de Vijver, 2012; van de Vijver \& Tanzer, 2004). Next, researchers should consider how constructs can be operationalized to be comparable across cultures. This could be partially accomplished by deductive, rational reasoning but is best empirically investigated through pilot studies. There are two general approaches to the empirical development of cross-culturally comparable instruments: (a) simultaneous development in multiple cultures to find common indicators (i.e., decentering); or (b) independent development within cultures and subsequent administration of all instruments in all cultures (i.e., convergence; He \& van de Vijver, 2012). In mobile sensing, decentering could mean collecting data from several cultures and identifying features that can be computed and constitute ecologically valid indicators of the target construct in every culture. Convergence might involve independently identifying and assessing construct-relevant behaviors using MSMs tailored to each culture, possibly using different sensor types and computing different features. In subsequent crosscultural studies, all of these culture-specific MSMs would then be used together. The

\footnotetext{
${ }^{4}$ In an optimal scenario, researchers would be able to a priori formulate a formalized generative causal modeling framework to determine what inferences can be drawn concerning generalizability (Deffner et al., 2021). However, given the complexity of models and the great number of variables in mobile sensing studies as well as a presumably greater focus on description and prediction (rather than explanation; see Harari, Vaid, et al., 2020, and Yarkoni and Westfall, 2017 for a detailed distinction), such an approach would not be feasible.
} 
convergence approach is considerably more arduous but allows for the examination of both universal and culture-specific aspects. However, it might not be possible to collect the same type of data in each culture, for example, due to heterogeneities in the sensing capabilities of devices (see Device-Type Bias) or local data protection laws (see Bias from Environmental Factors). Pilot studies should also include convergent measures of the target construct (e.g., questionnaires for which measurement invariance has already been demonstrated) as well as behavioral selfreports or observer reports. If behavioral ratings show differential associations with the target construct, they can be excluded from further investigations to avoid measurement bias (Tay et al., 2021). Lastly, researchers should clearly define a target population that exists in each culture and is comparable across cultures. Depending on whether researchers are mostly interested in cross-cultural similarities or differences, a diverse or homogenous subpopulation should be targeted (Boehnke et al., 2011; He \& van de Vijver, 2012). In smartphone sensing studies, researchers need to ensure that the target population is sufficiently represented among smartphone users in each culture and that smartphone ownership is not correlated with any variables (e.g., income) that may compromise sample comparability. If this is not the case, researchers may be forced to (a) define a narrower target population, which would reduce the within-culture generalizability of the findings; (b) exclude cultural groups in which the target population cannot be representatively sampled; or (c) attempt to statistically account for all potential covariates. In any case, researchers should ensure that relevant demographic variables and other theoretically relevant individual differences are assessed. Even when these variables are not intended to be included in the main analyses, they can be used to examine the comparability of cultural samples or to perform robustness checks. Furthermore, to mitigate possible biases from device-type demographics, researchers should determine the devices and 
operating systems typically used by the target population in each culture and create or select an inclusive sensing app, keeping in mind that "the range of supported devices should be as wide as possible, and at least reflect the targeted user groups in a representative manner" (Blunck et al., 2013, p. 1091).

The next stage concerns the implementation of the study, including the sampling and data collection processes. Participants are ideally selected from each culture such that the sample is representative of the predefined target population. Since sampling from the target population completely at random is rarely possible, researchers should attempt to make their recruitment efforts as inclusive as possible. Reaching certain demographics may require culture-specific recruiting strategies and incentives. For example, German senior citizens could be contacted via senior centers and nursing homes, whereas in China, it might be more effective to contact younger family members since intergenerational cohabitation is common. Local informants or collaborators can provide valuable information about how the target populations can be best approached. In addition, they can assist with properly translating and adapting study information and instructions. Researchers should ensure that participants are trained on each function and setting, are provided with examples and exercises where needed, and are encouraged to ask questions to assure that they fully understand the instructions. Investigators or research assistants who are in direct contact with participants should abide by standardized protocols and should optimally be trained in intercultural communication. To reduce biases from user practices, participants should also be clearly instructed on how to use the device during the data collection period (e.g., charging their phone regularly, always carrying it with them, etc.). If compliance with instruction is measurable, it can be explicitly incentivized. User practices cannot be fully standardized across cultural samples (e.g., participants cannot be obliged to carry their phones in 
their pockets). However, in some cases, it may be possible to assess individual differences in such practices through self-reports or estimates from sensor data for inclusion as categorical variables in analyses (e.g., in robustness checks). Biases from device properties can be mitigated by limiting functionality and sensing to what the more restrictive platforms and the less capable devices allow (Blunck et al., 2013). Researchers can also try to assess the performance of relevant sensors (for a validation approach, see Kayhan et al., 2018) or use archival data to account for differences between devices (Kos et al., 2016). Optimally, participants receive devices with identical, calibrated sensors, or the sensors embedded in their own devices can be auto-calibrated in the field (e.g., Altini et al., 2014; Sailhan et al., 2017). Lastly, because sensing software is rarely fully developed before data collection, researchers should expect that different software versions are needed to accommodate new requirements (e.g., to refine data collection, make use of previously unused sensors, etc.) or to debug. It is important to note that any software changes should be meticulously documented. Datasets from different versions can still be merged and used if they are adjusted accordingly based on documented changes (Blunck et al., 2013).

In the final, data-analytical stage, features are computed from raw sensor data and used for further analyses. As we have focused explicitly on biases that can occur at earlier stages and would already exist in raw sensor data, we will not go into significant detail here. Instead, we refer the reader to the relevant literature. Tay et al. (2021) proposed examining feature computing bias by correlating behavioral features from sensor data with behavioral observer rating. Differential associations between observer-rated behaviors and extracted features between cultures would indicate bias, and affected features should be excluded from further analyses. 
However, it should be kept in mind that observer rating can also harbor different biases and should be, thus, interpreted with caution.

The appropriate data-analytical approach depends on the study goal; conventional statistical approaches should be chosen to describe the data and make statistical inferences, while ML might be better suited to maximize prediction (Breiman, 2001; Yarkoni \& Westfall, 2017). When features computed from sensing data are treated as test items and analyzed with traditional descriptive and inferential statistical approaches, a wide range of modeling techniques are available to examine measurement invariance (e.g., differential item functioning analysis to detect item bias, multi-group CFAs, modeling subject and context variables, etc.; van de Vijver \& Tanzer, 2004). We refer the reader to the rich literature on measurement invariance testing (for overviews on the topic see, e.g., Tay et al., 2015; Teresi, 2006; van de Vijver \& Leung, 2021). However, traditional statistical methods may often not be suitable for mobile sensing data, for example, when there are large numbers of features but relatively few observations (i.e., participants; Orrù et al., 2020). For mitigation strategies concerning measurement bias in MLbased assessment, we refer the reader to Tay et al. (2021).

Lastly, from a meta-scientific perspective, it is important that researchers explicitly specify and report their targeted populations, contexts, and behavioral variables and indicate the degree of representativeness of their tested samples, situations (or situational variables), and behaviors (see Constraints on Generalizability statements; Simons et al., 2017). This practice would facilitate cumulative science, enabling other researchers to conduct direct replications and more targeted investigations of boundary conditions (Simons et al., 2017). From a cumulative science perspective, it would also be beneficial if mobile sensing researchers from computer science adopt some psychological reporting standards regarding, for example, sample 
description, materials, and descriptive statistics (e.g., Müller et al., in press provided a checklist of methodological information to report for GPS research).

\section{Limitations of Bias Mitigation Strategies}

The proposed mitigation strategies can minimize biases in cross-cultural mobile sensing research. However, in many cases, they might be challenging to implement. As mentioned before, biases in mobile sensing research can have cascading effects. This implies that biases must be circumvented or accounted for at each iterative stage of the research process to avoid their subsequent propagation. We recognize that this is time-consuming, labor-intensive, and cumbersome, and often not feasible. We also realize that many mobile sensing studies are not designed from scratch but use existing data. These studies need to work with the data they have but should be aware of, and also openly discuss, possible data heterogeneities and biases. Furthermore, mitigating biases often involves excluding participants (e.g., in distribution-based sample matching), measurement intervals, behavioral cues, or extracted features. While sequential exclusion may enhance comparability, there is a trade-off with validity. Specifically, limiting the representativeness of the sample and measurement contexts poses a threat to external validity. Excluding behavioral cues or computed features might lead to poor target construct coverage, affecting both content and construct validity.

One potential avenue to circumvent the issue of equivalence is to abandon its necessity in the first place. To do this, we need to refocus the aim of our research to generalize within cultures instead of between cultures. As such, nested analyses (such as multilevel models) can allow for teasing apart the effects and relationships that only hold within a specific culture compared to those that generalize across the cultures included in the model. 


\section{Conclusion}

Mobile sensing can be used to assess people's real-life behaviors objectively, unobtrusively, and with high temporal resolutions. It might be especially suited for cross-cultural research since it does not use verbal items that need to be adapted into different languages - and inappropriate instrument translation or insufficient adaptation to different cultural contexts are the roots of many biases that can jeopardize cross-cultural comparisons. Furthermore, as smartphone ownership continues to increase worldwide, smartphone sensing research might be able to sample subpopulations that would not typically participate in psychological research, thus promoting more diverse and inclusive research. However, to date, only very few cross-cultural mobile sensing studies have been conducted, and multi-country studies often fail to acknowledge or examine possible cross-cultural differences. In this chapter, we illustrated different biases that can occur when conducting cross-cultural mobile sensing studies and proposed mitigation strategies to minimize these biases. We believe that more and especially rigorous comparative research is needed to establish and refine MSMs for cross-cultural psychology. Therefore, we encourage cross-cultural researchers to make use of mobile sensing approaches, which may prove to be an invaluable addition to their methodological toolkit. 


\section{References}

Ai, P., Liu, Y., \& Zhao, X. (2019). Big Five personality traits predict daily spatial behavior:

Evidence from smartphone data. Personality and Individual Differences, 147, 285-291. https://doi.org/10.1016/j.paid.2019.04.027

Åkerberg, A., Lindén, M., \& Folke, M. (2012). How accurate are pedometer cell phone applications? Procedia Technology, 5, 787-792.

https://doi.org/10.1016/j.protcy.2012.09.087

Altini, M., Vullers, R., Van Hoof, C., van Dort, M., \& Amft, O. (2014, March). Self-calibration of walking speed estimations using smartphone sensors. In 2014 IEEE International Conference on Pervasive Computing and Communication Workshops (PERCOM WORKSHOPS) (pp. 10-18). IEEE. https://doi.org/10.1109/PerComW.2014.6815158

Bai, Y., Xu, B., Ma, Y., Sun, G., \& Zhao, Y. (2012). Will you have a good sleep tonight? Sleep quality prediction with mobile phone. In I. Balasingham (Ed.), Proceedings of the $7^{\text {th }}$ International Conference on Body Area Networks (pp. 124-130). Institute for Computer Sciences, Social-Informatics and Telecommunications Engineering. https://doi.org/10.4108/icst.bodynets.2012.250091

Bastos, A. S., \& Hasegawa, H. (2013). Behavior of GPS signal interruption probability under tree canopies in different forest conditions. European Journal of Remote Sensing, 46(1), 613-622. https://doi.org/10.5721/eujrs20134636

Bauer, D. J. (2017). A more general model for testing measurement invariance and differential item functioning. Psychological Methods, 22(3), 507.

https://doi.org/10.1037/met0000077 
Blunck, H., Bouvin, N. O., Franke, T., Grønbæk, K., Kjaergaard, M. B., Lukowicz, P., \& Wüstenberg, M. (2013). On heterogeneity in mobile sensing applications aiming at representative data collection. In F. Mattern (Ed.), Proceedings of the 2013 ACM Conference on Pervasive and Ubiquitous Computing Adjunct Publication (pp. 10871098). Association for Computing Machinery. https://doi.org/10.1145/2494091.2499576

Boehnke, K., Lietz, P., Schreier, M., \& Wilhelm, A. (2011). Sampling: The selection of cases for culturally comparative psychological research. In D. Matsumoto \& F. J. R. van de Vijver (Eds.), Cross-cultural research methods in psychology (pp. 101-129). Cambridge University Press.

Borsboom, D. (2006). When does measurement invariance matter?. Medical Care, 44(11), S176S181. https://doi.org/10.1097/01.mlr.0000245143.08679.cc

Borsboom, D., Romeijn, J. W., \& Wicherts, J. M. (2008). Measurement invariance versus selection invariance: Is fair selection possible?. Psychological Methods, 13(2), 75. https://doi.org/10.1037/1082-989X.13.2.75

Breiman, L. (2001). Statistical modeling: The two cultures (with comments and a rejoinder by the author). Statistical Science, 16(3), 199-231. https://doi.org/10.1214/ss/1009213726

Brunswik, E. (1956). Perception and the representative design of psychological experiments $\left(2^{\text {nd }}\right.$ ed.). University of California Press.

Byrne, B. M., \& van de Vijver, F. (2010). Testing for measurement and structural equivalence in large-scale cross-cultural studies: Addressing the issue of nonequivalence. International Journal of Testing, 10(2), 107-132. https://doi.org/10.1080/15305051003637306 
Chaffin, D., Heidl, R., Hollenbeck, J. R., Howe, M., Yu, A., Voorhees, C., \& Calantone, R. (2017). The promise and perils of wearable sensors in Organizational Research. Organizational Research Methods, 20(1), 3-31. https://doi.org/10.1177/1094428115617004

Cornet, V. P., \& Holden, R. J. (2018). Systematic review of smartphone-based passive sensing for health and wellbeing. Journal of Biomedical Informatics, 77, 120-132. https://doi.org/10.1016/j.jbi.2017.12.008

Counterpoint. (2021, September 13). Top 5 smartphone model share for 8 countries. https://www.counterpointresearch.com/top-5-smartphone-model-share-8-countries/

de Vries, L. P., Baselmans, B. M., \& Bartels, M. (2021). Smartphone-based ecological momentary assessment of well-being: A systematic review and recommendations for future studies. Journal of Happiness Studies, 22(5), 2361-2408. https://doi.org/10.1007/s10902-020-00324-7

Deffner, D., Rohrer, J. M., \& McElreath, R. (2021). A causal framework for cross-cultural generalizability. PsyAirXiv. https://doi.org/10.31234/osf.io/fqukp

Delaporte, A., Bahia, K., Carboni, I., Cruz, G., Jeffrie, N., Sibthorpe, C., Suardi, S., \& Groenestege, M. T. (2021). The state of mobile internet connectivity 2021. GSM Association. https://www.gsma.com/r/wp-content/uploads/2021/09/The-State-of-MobileInternet-Connectivity-Report-2021.pdf

Grammenos, A., Mascolo, C., \& Crowcroft, J. (2018). You are sensing, but are you biased? Proceedings of the ACM on Interactive, Mobile, Wearable and Ubiquitous Technologies, 2(1), 1-26. https://doi.org/10.1145/3191743 
Harari, G. M., Gosling, S. D., Wang, R., Chen, F., Chen, Z., \& Campbell, A. T. (2017). Patterns of behavior change in students over an academic term: A preliminary study of activity and sociability behaviors using smartphone sensing methods. Computers in Human Behavior, 67, 129-138. https://doi.org/10.1016/j.chb.2016.10.027

Harari, G. M., Lane, N. D., Wang, R., Crosier, B. S., Campbell, A. T., \& Gosling, S. D. (2016). Using smartphones to collect behavioral data in psychological science. Perspectives on Psychological Science, 11(6), 838-854. https://doi.org/10.1177/1745691616650285

Harari, G. M., Müller, S. R., Aung, M. S. H., \& Rentfrow, P. J. (2017). Smartphone sensing methods for studying behavior in everyday life. Current Opinion in Behavioral Sciences, 18, 83-90. https://doi.org/10.1016/j.cobeha.2017.07.018

Harari, G. M., Müller, S. R., \& Gosling, S. D. (2020). Naturalistic assessment of situations using mobile sensing methods. In J. F. Rauthmann, R. A. Sherman, \& D. C. Funder (Eds.), The Oxford handbook of psychological situations (pp. 299-311). Oxford University Press.

Harari, G. M., Müller, S. R., Stachl, C., Wang, R., Wang, W., Bühner, M., Rentfrow, P. J., Campbell, A. T., \& Gosling, S. D. (2020). Sensing sociability: Individual differences in young adults' conversation, calling, texting, and app use behaviors in daily life. Journal of Personality and Social Psychology, 119(1), 204-228.

\section{https://doi.org/10.1037/pspp0000245}

Harari, G. M., Stachl, C., Müller, S. R., \& Gosling, S. D. (2021). Mobile sensing for studying personality dynamics in daily life. In J. F. Rauthmann (Ed.), The handbook of personality dynamics and processes (pp. 763-790). Academic Press. https://doi.org/10.1016/B978-0-

\section{$\underline{12-813995-0.00029-7}$}


Harari, G. M., Vaid, S. S., Müller, S. R., Stachl, C., Marrero, Z., Schoedel, R., Bühner, M., \& Gosling, S. D. (2020). Personality sensing for theory development and assessment in the Digital age. European Journal of Personality, 34(5), 649-669. https://doi.org/10.1002/per.2273

He, J., \& van de Vijver, F. (2012). Bias and equivalence in cross-cultural research. Online Readings in Psychology and Culture, 2(2), 1-19. https://doi.org/10.9707/2307-0919.1111

Henrich, J., Heine, S. J., \& Norenzayan, A. (2010a). Beyond weird: Towards a broad-based behavioral science. Behavioral and Brain Sciences, 33(2-3), 111-135. https://doi.org/10.1017/s0140525x10000725

Henrich, J., Heine, S. J., \& Norenzayan, A. (2010b). The weirdest people in the world?. Behavioral and Brain Sciences, 33(2-3), 61-83. https://doi.org/10.1017/S0140525X0999152X

Horstmann, K. T., \& Ziegler, M. (2020). Assessing personality states: What to consider when constructing personality state measures. European Journal of Personality, 34(6), 10371059. https://doi.org/10.1002/per.2266

John, O. P., \& Robins, R. W. (1993). Determinants of interjudge agreement on personality traits: The Big Five Domains, Observability, evaluativeness, and the unique perspective of the self. Journal of Personality, 61(4), 521-551. https://doi.org/10.1111/j.14676494.1993.tb00781.x

Kayhan, V. O., Chen, Z., French, K. A., Allen, T. D., Salomon, K., \& Watkins, A. (2018). How honest are the signals? A protocol for validating wearable sensors. Behavior Research Methods, 50(1), 57-83. https://doi.org/10.3758/s13428-017-1005-4 
Khan, W. Z., Xiang, Y., Aalsalem, M. Y., \& Arshad, Q. (2012). Mobile phone sensing systems: A survey. IEEE Communications Surveys \& Tutorials, 15(1), 402-427. https://doi.org/10.1109/SURV.2012.031412.00077

Khwaja, M., Vaid, S. S., Zannone, S., Harari, G. M., Faisal, A. A., \& Matic, A. (2019). Modeling personality vs. modeling personalidad: In-the-wild mobile data analysis in five countries suggests cultural impact on personality models. Proceedings of the ACM on Interactive, Mobile, Wearable and Ubiquitous Technologies, 3(3), 1-24. https://doi.org/10.1145/3351246

Killingsworth, M. A., \& Gilbert, D. T. (2010). A wandering mind is an unhappy mind. Science, 330(6006), 932-932. https://doi.org/10.1126/science.1192439

Kos, A., Tomažič, S., \& Umek, A. (2016). Evaluation of smartphone inertial sensor performance for cross-platform mobile applications. Sensors, 16(4), 1-15. https://doi.org/10.3390/s16040477

Krendl, A. C., \& Pescosolido, B. A. (2020). Countries and Cultural Differences in the Stigma of Mental Illness: The East-West Divide. Journal of Cross-Cultural Psychology, 51(2), 149-167. https://doi.org/10.1177/0022022119901297

Kuhlmann, T., Garaizar, P., \& Reips, U.-D. (2020). Smartphone sensor accuracy varies from device to device in mobile research: The case of spatial orientation. Behavior Research Methods, 53(1), 22-33. https://doi.org/10.3758/s13428-020-01404-5

Lane, N. D., Mohammod, M., Lin, M., Yang, X., Lu, H., Ali, S., ... \& Campbell, A. (2011, May). Bewell: A smartphone application to monitor, model and promote wellbeing. In 5th 
international ICST conference on pervasive computing technologies for healthcare (pp. 23-26).

Lantz, B. (2019). Machine learning with R: Expert techniques for predictive modeling ( $3^{\text {rd }}$. ed.). Packt Publishing.

Lathia, N., Rachuri, K. K., Mascolo, C., \& Rentfrow, P. J. (2013). Contextual dissonance: Design bias in sensor-based experience sampling methods. In F. Mattern (Ed.), Proceedings of the 2013 ACM International Joint Conference on Pervasive and Ubiquitous Computing (pp. 183-192). Association for Computing Machinery. https://doi.org/10.1145/2493432.2493452

Lee, H., Ahn, H., Choi, S., \& Choi, W. (2014). The SAMS: Smartphone Addiction Management System and verification. Journal of Medical Systems, 38(1), 1-10. https://doi.org/10.1007/s10916-013-0001-1

Leong, J. Y., \& Wong, J. E. (2016). Accuracy of three Android-based pedometer applications in laboratory and free-living settings. Journal of Sports Sciences, 35(1), 14-21. https://doi.org/10.1080/02640414.2016.1154592

Ma, Y., Xu, B., Bai, Y., Sun, G., \& Zhu, R. (2012). Daily mood assessment based on mobile phone sensing. In G.-Z. Yang (Ed.), 2012 Ninth International Conference on Wearable and Implantable Body Sensor Networks (pp. 142-147). Institute of Electrical and Electronics Engineers. https://doi.org/10.1109/bsn.2012.3

Ma, L., Zhang, C., Wang, Y., Peng, G., Chen, C., Zhao, J., \& Wang, J. (2020). Estimating urban road GPS environment friendliness with bus trajectories: A city-scale approach. Sensors, 20(6), 1580. https://doi.org/10.3390/s20061580 
Meredith, W. (1993). Measurement invariance, factor analysis and factorial invariance. Psychometrika, 58(4), 525-543. https://doi.org/10.1007/BF02294825

Mohr, D. C., Zhang, M., \& Schueller, S. M. (2017). Personal sensing: Understanding mental health using ubiquitous sensors and machine learning. Annual Review of Clinical Psychology, 13(1), 23-47. https://doi.org/10.1146/annurev-clinpsy-032816-044949

Müller, S. R., Bayer, J. B., Ross, M. Q., Mount, J., Stachl, C., Harari, G. M., Chang, Y.-J., Le, H. (in press). Analyzing GPS Data for Psychological Research: A Tutorial. Advances in Methods and Practices in Psychological Science. https://doi.org/10.31234/osf.io/3cq8n

Müller, S. R., Chen, X. L., Peters, H., Chaintreau, A., \& Matz, S. C. (2021). Depression predictions from GPS-based mobility do not generalize well to large demographically heterogeneous samples. Scientific Reports, 11, 1-10. https://doi.org/10.1038/s41598-021$\underline{93087-\mathrm{X}}$

Müller, S. R., Peters, H., Matz, S. C., Wang, W., \& Harari, G. M. (2020). Investigating the relationships between mobility behaviours and indicators of subjective well-being using smartphone-based experience sampling and GPS tracking. European Journal of Personality, 34(5), 714-732. https://doi.org/10.1002\%2Fper.2262

Oort, F. J., Visser, M. R., \& Sprangers, M. A. (2009). Formal definitions of measurement bias and explanation bias clarify measurement and conceptual perspectives on response shift. Journal of Clinical Epidemiology, 62(11), 1126-1137.

https://doi.org/10.1016/j.jclinepi.2009.03.013 
Orrù, G., Monaro, M., Conversano, C., Gemignani, A., \& Sartori, G. (2020). Machine learning in Psychometrics and psychological research. Frontiers in Psychology, 10, 1-10. https://doi.org/10.3389/fpsyg.2019.02970

Pernot-Leplay, E. (2020). China's approach on data privacy law: A third way between the US and the EU? Penn State Journal of Law \& International Affairs, 8(1), 49-117. https://elibrary.law.psu.edu/jlia/vol8/iss 1/6

Phan, L. V., \& Rauthmann, J. F. (2021). Personality computing: New frontiers in personality assessment. Social and Personality Psychology Compass, 15(7), 1-17. https://doi.org/10.1111/spc3.12624

Putnick, D. L., \& Bornstein, M. H. (2016). Measurement invariance conventions and reporting: The state of the art and future directions for psychological research. Developmental Review, 41, 71-90. https://doi.org/10.1016/j.dr.2016.06.004

Rad, M. S., Martingano, A. J., \& Ginges, J. (2018). Toward a psychology of Homo sapiens: Making psychological science more representative of the human population. Proceedings of the National Academy of Sciences, 115(45), 11401-11405. https://doi.org/10.1073/pnas.1721165115

Rabbi, M., Ali, S., Choudhury, T., \& Berke, E. (2011, September 17-21). Passive and in-situ assessment of mental and physical well-being using mobile sensors. In J. Landay \& Y. Shi (Chairs), How healthy? [Symposium]. Proceedings of the $13^{\text {th }}$ International Conference on Ubiquitous Computing, Beijing, China. https://doi.org/10.1145/2030112.2030164 
Ram, N., Conroy, D. E., Pincus, A. L., Lorek, A., Rebar, A., Roche, M. J., Coccia, M., Morack, J., Feldman, J., \& Gerstorf, D. (2014). Examining the interplay of processes across multiple time-scales: Illustration with the intraindividual study of affect, health, and interpersonal behavior (isahib). Research in Human Development, 11(2), 142-160. https://doi.org/10.1080/15427609.2014.906739

Rauthmann, J. F. (2016). Motivational factors in the perception of psychological situation characteristics. Social and Personality Psychology Compass, 10(2), 92-108. https://doi.org/10.1111/spc3.12239

Rauthmann, J. F. (2021). Capturing interactions, correlations, fits, and transactions: A personenvironment relations model. In J. F. Rauthmann (Ed.), The handbook of personality dynamics and processes (pp. 427-522). Academic Press. https://doi.org/10.1016/b978-0$12-813995-0.00018-2$

R Core Team (2021). R: A language and environment for statistical computing. R Foundation for Statistical Computing, Vienna, Austria. https://www.R-project.org/.

Sailhan, F., Issarny, V. \& Tavares-Nascimiento, O. (2017, October). Opportunistic multiparty calibration for robust participatory sensing. In 2017 IEEE $14^{\text {th }}$ International Conference on Mobile Ad Hoc and Sensor Systems (MASS) (pp. 435-443). IEEE. https://doi.org/10.1109/MASS.2017.56

Sanchez, W., Martinez, A., Campos, W., Estrada, H., \& Pelechano, V. (2015). Inferring loneliness levels in older adults from smartphones. Journal of Ambient Intelligence and Smart Environments, 7(1), 85-98. https://doi.org/10.3233/ais-140297 
Sandstrom, G. M., Lathia, N., Mascolo, C., \& Rentfrow, P. J. (2017). Putting mood in context: Using smartphones to examine how people feel in different locations. Journal of Research in Personality, 69, 96-101. https://doi.org/10.1016/j.jrp.2016.06.004

Schoedel, R., Pargent, F., Au, Q., Völkel, S. T., Schuwerk, T., Bühner, M., \& Stachl, C. (2020). To challenge the morning lark and the night owl: Using smartphone sensing data to investigate day-night behaviour patterns. European Journal of Personality, 34(5), 733752. https://doi.org/10.1002/per.2258

Silver, L. (2019, February 5). Smartphone ownership is growing rapidly around the world, but not always equally. Pew Research Center.

https://www.pewresearch.org/global/2019/02/05/smartphone-ownership-isgrowing-rapidly-around-the-world-but-not-always-equally/

Simons, D. J., Shoda, Y., \& Lindsay, D. S. (2017). Constraints on Generality (COG): A proposed addition to all empirical papers. Perspectives on Psychological Science, 12(6), 11231128. https://doi.org/10.1177/1745691617708630

Stachl, C., Au, Q., Schoedel, R., Gosling, S. D., Harari, G. M., Buschek, D., Völkel, S. T., Schuwerk, T., Oldemeier, M., Ullmann, T., Hussmann, H., Bischl, B., \& Bühner, M. (2020). Predicting personality from patterns of behavior collected with smartphones. Proceedings of the National Academy of Sciences of the United States of America, 117(30), 17680-17687. https://doi.org/10.1073/pnas.1920484117

Stachl, C., Pargent, F., Hilbert, S., Harari, G. M., Schoedel, R., Vaid, S., Gosling, S. D., \& Bühner, M. (2020). Personality research and assessment in the era of machine learning. European Journal of Personality, 34(5), 613-631. https://doi.org/10.1002/per.2257 
Statista. (2020, August 20). Number of smartphone users from 2016 to 2021.

https://www.statista.com/statistics/330695/number-of-smartphone-users-worldwide/

Stieger, S., Götz, F. M., \& Gehrig, F. (2015). Soccer results affect subjective well-being, but only briefly: A smartphone study during the 2014 FIFA World Cup. Frontiers in Psychology, 6. https://doi.org/10.3389/fpsyg.2015.00497

Tay, L., Meade, A. W., \& Cao, M. (2014). An overview and practical guide to IRT measurement equivalence analysis. Organizational Research Methods, 18(1), 3-46. https://doi.org/10.1177/1094428114553062

Tay, L., Woo, S. E., Hickman, L., Booth, B. M., \& D’Mello, S. (2021). A conceptual framework for investigating and mitigating Machine Learning Measurement Bias (MLMB) in psychological assessment. PsyArXiv. https://doi.org/10.31234/osf.io/mjph3

Tennekes M (2018). Tmap: Thematic Maps in R. Journal of Statistical Software, 84(6), 1-39. https://doi.org/10.18637/jss.v084.i06

Teresi, J. A. (2006). Overview of quantitative measurement methods: Equivalence, invariance, and differential item functioning in health applications. Medical Care, 44(11), S39-S49. https://doi.org/10.1097/01.mlr.0000245452.48613.45

Vaid, S., \& Harari, G. M. (2019). Smartphones in Personal Informatics: A Framework for SelfTracking Research with Mobaile Sensing,. In H. Baumeister, Montag, C. (Ed.), The Digital Phenotyping an Mobile Sensing: Studies in Neuroscience, Psychology and Behavioral Economics. Springer. https://doi.org/10.1007/978-3-030-31620-4_5

van de Vijver, F., \& Leung, K. (2021). Methods and data analysis for cross-cultural research (2nd ed.). Cambridge University Press. 
van de Vijver, F., \& Tanzer, N. K. (2004). Bias and equivalence in cross-cultural assessment: An overview. European Review of Applied Psychology, 54(2), 119-135. https://doi.org/10.1016/j.erap.2003.12.004

Vinciarelli, A., \& Mohammadi, G. (2014). A survey of personality computing. IEEE Transactions on Affective Computing, 5(3), 273-291. https://doi.org/10.1109/TAFFC.2014.2330816

von Stumm, S. (2018). Feeling low, thinking slow? associations between situational cues, mood and cognitive function. Cognition and Emotion, 32(8), 1545-1558. https://doi.org/10.1080/02699931.2017.1420632

Wahl, D. R., Villinger, K., König, L. M., Ziesemer, K., Schupp, H. T., \& Renner, B. (2017). Healthy Food Choices are happy food choices: Evidence from a real life sample using smartphone based assessments. Scientific Reports, 7(1). https://doi.org/10.1038/s41598017-17262-9

Wang, R., Chen, F., Chen, Z., Li, T., Harari, G., Tignor, S., Zhou, X., Ben-Zeev, D., \&. Campbell, A. T. (2014, September 13-17). StudentLife: Assessing mental health, academic performance and behavioral trends of college students using smartphones. In A. J. Brush (Ed.), Proceedings of the 2014 ACM International Joint Conference on Pervasive and Ubiquitous Computing (pp. 3-14). Association for Computing Machinery. https://doi.org/10.1145/2632048.2632054

Wang, W., Harari, G. M., Wang, R., Müller, S. R., Mirjafari, S., Masaba, K., \& Campbell, A. T. (2018). Sensing behavioral change over time: Using within-person variability features from mobile sensing to predict personality traits. Proceedings of the ACM on Interactive, 
Mobile, Wearable and Ubiquitous Technologies, 2(3), 1-21.

https://doi.org/10.1145/3264951

Wiernik, B. M., Ones, D. S., Marlin, B. M., Giordano, C., Dilchert, S., Mercado, B. K., Stanek, K. C., Birkland, A., Wang, Y., Ellis, B., Yazar, Y., Kostal, J. W., Kumar, S., Hnat, T., Ertin, E., Sano, A., Ganesan, D. K., Choudhoury, T., \& Al'Absi, M. (2020). Using mobile sensors to study personality dynamics. European Journal of Psychological Assessment, 36(6), 1-13. https://doi.org/10.1027/1015-5759/a000576

Woo, S. E., Tay, L., Jebb, A. T., Ford, M. T., \& Kern, M. L. (2020). Big data for enhancing measurement quality. In S. E. Woo, L. Tay, \& R. W. Proctor (Eds.), Big data in psychological research (pp. 59-85). American Psychological Association. https://doi.org/10.1037/0000193-004

Yan, Z., \& Chakraborty, D. (2014). Semantics in mobile sensing. Morgan \& Claypool. https://doi.org/10.2200/S00577ED1V01Y201404WBE008

Yarkoni, T., \& Westfall, J. (2017). Choosing prediction over explanation in psychology: Lessons from machine learning. Perspectives on Psychological Science, 12(6), 1100-1122. https://doi.org/10.1177/1745691617693393

Zhang, X., Li, W., Chen, X., \& Lu, S. (2018). MoodExplorer: Towards compound emotion detection via smartphone sensing. Proceedings of the ACM on Interactive, Mobile, Wearable and Ubiquitous Technologies, 1(4), 1-30. https://doi.org/10.1145/3161414 